\title{
Efeito do beneficiamento sobre o valor nutricional do peixe mandim (Arius spixii)
}

\author{
Fabiana Rodrigues de Oliveira ${ }^{1}$, Giselda Macena Liraa ${ }^{1 *}$, Elizabeth Aparecida Ferraz da Silva Torres ${ }^{2}$, \\ Rosana Aparecida Manólio Soares ${ }^{2}$, Simone Mendonça ${ }^{2}$, Kelly Walkyria Barros da Silva ${ }^{1}$, Sarah \\ Janaína Gurgel Bechtinger Simon', Tatiana Maria Palmeira dos Santos', Cyro Rego Cabral Junior ${ }^{1}$
}

${ }^{1}$ Faculdade de Nutrição, Universidade Federal de Alagoas, ${ }^{2}$ Departamento de Nutrição, Faculdade de Saúde Pública, Universidade de São Paulo

*Correspondência:

G. M Lira

Faculdade de Nutrição

Universidade Federal de Alagoas

BR 101, Km 14

57.072-900 - Maceió - AL, Brasil

E-mail: gmlira@ig.com.br
Com o objetivo de avaliar o efeito do beneficiamento sobre o valor nutricional do peixe mandim (Arius spixii) comercializado em Maceió-AL, determinaram-se nas suas formas in natura e beneficiada (salgado-seco) a composição centesimal, valor calórico, cloretos, perfil de ácidos graxos e colesterol, sendo também analisada a ocorrência de óxidos de colesterol. Os resultados obtidos para o mandim in natura e beneficiado, respectivamente, de umidade (70,13\% e 40,31\%), proteinas $(51,73 \%$ e $38,07 \%$, base seca), carboidratos (4,67\% e 2,24\%, base seca), calorias (486 kcal/100g e $367 \mathrm{kcal} / 100 \mathrm{~g}$, base seca), ácidos graxos (poliinsaturados 14,54\% e 15,49\%, ômega-3 8,51\% e 6,51\%), colesterol (82,66 mg/100g e $61,30 \mathrm{mg} / 100 \mathrm{~g}$ ) e óxidos (7-cetocolesterol $8,31 \mu \mathrm{g} / \mathrm{g}$ e 17,90 $\mu \mathrm{g} / \mathrm{g}$ ), permitiram concluir que o beneficiamento favoreceu alterações significativas no valor nutricional do mandim.
Unitermos

- Peixe/valor nutricional

- Pescado/beneficiamento

- Pescado/composição centesimal

- Cloretos/determinação em pescado

- Ácidos graxos/ determinação em pescado

- Óxidos de colesterol/ determinação em pescado

\section{INTRODUÇÃO}

A cada dia buscam-se tecnologias que objetivem melhor aproveitamento do pescado, pelo fato de ser um alimento altamente perecível quando in natura, susceptível à contaminação microbiológica e a escasso tempo de vida útil (Jesus et al., 2002).

Com certa tradição em algumas regiões brasileiras, a salga é um método muito empregado na conservação de produtos cárneos, sendo de grande importância onde a refrigeração torna-se difícil (Furtado et al., 1991). Dentre o pescado destinado à elaboração de produtos salgados no Brasil, encontra-se o peixe mandim (Arius spixii), cujo consumo é popular na região do entorno do Complexo Estuarino das Lagoas Mundaú/Manguaba, Estado de Alagoas.
O mandim é um tipo de bagre pequeno, com cerca de $30 \mathrm{~cm}$ de comprimento, muito comum no Complexo Mundaú/Manguaba. Não se trata de um peixe de valor comercial, mas sim de grande valor social, devido ao seu baixo custo. É comercializado logo após a pesca e após beneficiamento, no local de desembarque e nas feiras livres. O beneficiamento consiste em evisceração, salga seca e secagem ao sol (Costa, 1980).

A salga é um processo de concentração no qual o sal (cloreto de sódio) é a substância química utilizada e, pelas suas propriedades físicas e físico-químicas de deliqüescência e higroscopicidade, é o agente desidratante. Pode ser efetuada por via seca, úmida (com uso de salmoura), ou ainda, por combinação das duas vias (salga mista). Para garantir melhor conservação do produto, na finalização da salga costuma-se empregar processos com- 
plementares como secagem e/ou refrigeração (Baruffaldi, Oliveira, 1998).

A secagem pode ser efetuada ao sol ou através do uso de secadores industriais, em temperatura superior a $30{ }^{\circ} \mathrm{C}$, e por tempo suficiente para a redução de umidade e atividade de água (Bastos, 1977).

Apesar do pescado salgado-seco ser considerado um produto estável, uma vez que a diminuição da concentração de água inibiria a ocorrência de reações químicas e desenvolvimento microbiano, ele não está livre de sofrer deterioração, seja ela química ou microbiológica, principalmente se falhas ao longo do processo ocorrerem (Morais, Silveira, 1994).

A exposição de produtos cárneos salgados ao calor pode provocar alterações na sua composição química (Furtado et al., 1992). Associado ao calor, o sal torna-se um forte pró-oxidante das gorduras, ativando a lipoxidase do músculo (Pardi et al., 2001). No pescado, essa degradação é proveniente principalmente da oxidação de ácidos graxos poliinsaturados (Cornejo, Nogueira, Park, 1997).

Os peixes representam a maior fonte dietética de ácidos graxos poliinsaturados, especialmente os da série ômega-3 (Kotb, Hadeed, Al-Baker, 1991; Sargent, Henderson, 1995), aos quais se atribuem numerosos benefícios ao organismo humano (Herold, Kinsella, 1986; Burr, 1989; Sargent, 1997).

A qualidade do produto final, salgado-seco, depende fundamentalmente do estado da matéria-prima e da utilização de técnicas corretas (Nort, 1974 apud Reale, 1997). O efeito do processamento pode ser o fator primário, por exemplo, para a oxidação do colesterol em peixes secos (Chen, Yeh, 1994).

Os óxidos de colesterol (OsC) são substâncias biologicamente ativas, associadas a processos citotóxicos, aterogênicos, mutagênicos e cancerígenos (MoralesAizpurúa e Tenuta-Filho, 2005). Os mais comuns em alimento são: 7-cetocolesterol (7-Ceto), 20-hidroxicolesterol (20-OH), 25-hidroxicolesterol (25-OH), $7 \alpha$-hidroxicolesterol $(7 \alpha-\mathrm{OH}), 7 \beta$-hidroxicolesterol $(7 \beta-\mathrm{OH})$,

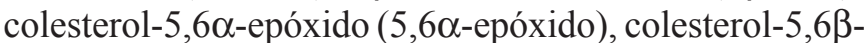
epóxido (5,6ß-epóxido) e o colestanotriol (Triol) (Tai, Chen, Chen, 1999).

Considerando que o peixe mandim (Arius spixii) comercializado em Maceió-AL é beneficiado de forma totalmente empírica, procedida sem técnica e critérios, este trabalho teve como objetivo avaliar o efeito do beneficiamento sobre o seu valor nutricional, visando a inclusão de alimento nunca antes analisado e de grande valor social em Tabelas de Composição Química Regionais/Nacionais, o conhecimento das implicações para a saúde do seu consumo, e o fornecimento de subsídios para aperfeiçoamento do processo tecnológico artesanal ao qual ele é submetido.

\section{MATERIAL E MÉTODOS}

\section{Amostragem}

Foram analisadas 30 amostras de peixe mandim (Arius spixii), procedentes do Complexo Estuarino das Lagoas Mundaú/Manguaba, distribuídas da seguinte forma: 15 in natura, coletadas logo após a pesca, e 15 beneficiadas, coletadas imediatamente após o processamento. $\mathrm{O}$ beneficiamento consiste em evisceração, salga seca e secagem ao sol (Alagoas, 1980). As amostras foram adquiridas no local de desembarque da pesca, em Maceió-AL, no período de 30 de abril a 31 de maio de 2006 , em lotes de $1,2 \mathrm{~kg}$ (mandim in natura) e $1,0 \mathrm{~kg}$ (mandim beneficiado), com animais com peso médio de $40 \mathrm{~g}$ cada.

\section{Preparo das amostras}

Imediatamente após a coleta, os lotes foram acondicionados em sacos plásticos esterilizados, dispostos em caixas de isopor com gelo, e conduzidos ao Laboratório de Bromatologia da Faculdade de Nutrição da Universidade Federal de Alagoas. De cada lote, foram tomados, em média, $200 \mathrm{~g}$ de peixe para preparo das amostras, havendo previamente evisceração e limpeza dos animais in natura. As amostras foram novamente embaladas em sacos plásticos esterilizados, identificadas e congeladas a $-17^{\circ} \mathrm{C}$ até o momento da realização das análises.

\section{Determinações analíticas}

Após homogeneização da amostra, foram realizadas as seguintes determinações em duplicata:

\section{Composição centesimal}

Umidade - determinada gravimetricamente em estufa a $105^{\circ} \mathrm{C}$ (AOAC, 1990).

Cinzas - obtidas por incineração em mufla a $550^{\circ} \mathrm{C}$ (AOAC, 1990).

Proteínas - determinadas pelo método Kjedahl. O fator de conversão da percentagem de nitrogênio para proteína bruta foi 6,25 (AOAC, 1990).

Lipídios totais - extraídos a frio pelo método de Folch, Lees e Sloanne Stanley (1957). Alíquotas foram tomadas para determinações gravimétricas.

Carboidratos - quantificados por diferença, através da subtração dos percentuais de umidade, cinzas, proteínas e lipídios da percentagem total (100\%). 


\section{Valor calórico}

Calculado a partir dos coeficientes calóricos correspondentes para proteínas, carboidratos e lipídeos: 4, 4 e 9 $\mathrm{kcal} / \mathrm{g}$, respectivamente (Brasil, 1998).

\section{Cloretos}

Determinados por volumetria (Instituto Adolfo Lutz, 2005).

\section{Perfil de ácidos graxos}

Os extratos lipídicos obtidos pelo método de Folch, Lees e Sloanne Stanley (1957) foram esterificados segundo Hartman e Lago (1973), visando à determinação dos ésteres metílicos de ácidos graxos por cromatografia gasosa, e encaminhados ao Laboratório de Bromatologia do Departamento de Nutrição da Faculdade de Saúde Pública da Universidade de São Paulo. Para a identificação dos ácidos graxos foram utilizados padrões de ésteres metílicos de ácidos graxos (37 FAME Mix 47885, Supelco). Os resultados foram expressos em percentual de área de cada pico sobre o total de ácidos graxos.

Os parâmetros de operação do equipamento estão relatados a seguir: cromatógrafo a gás Chrompack $\mathrm{CP}$ 9002 (Middelburg, Holanda), equipado com coluna capilar CiolaWax de 20 metros, diâmetro de $0,32 \mathrm{~mm}$ e espessura do filme $0,25 \mu \mathrm{m}$. O forno foi operado com temperatura inicial de $60{ }^{\circ} \mathrm{C}$ por $2 \mathrm{~min}$, e a rampa de temperatura foi de $4{ }^{\circ} \mathrm{C} / \mathrm{min}$ até atingir $141{ }^{\circ} \mathrm{C}, 3,5^{\circ} \mathrm{C} / \mathrm{min}$ até $176^{\circ} \mathrm{C}$, $2{ }^{\circ} \mathrm{C} /$ min até atingir $186^{\circ} \mathrm{C}$, e $3,5^{\circ} \mathrm{C} / \mathrm{min}$ até atingir a temperatura final de $240{ }^{\circ} \mathrm{C}$. A temperatura do injetor era de $270{ }^{\circ} \mathrm{C}$ e do detector de $300^{\circ} \mathrm{C}$. O gás de arraste utilizado foi o hidrogênio, com fluxo de $1,9 \mathrm{~mL} / \mathrm{min}$, razão de divisão 1:30. Foi injetado um volume de $1 \mu \mathrm{L}$ de amostra.

\section{Colesterol e óxidos de colesterol $(\mathrm{OsC})$ livres: 7-Ceto, $7 \alpha-\mathrm{OH}, 7 \beta-\mathrm{OH}$ e $25-\mathrm{OH}$}

Extraídos pelo método de Csallany e Ayaz (1976), adaptado de Folch, Lees e Sloanne Stanley (1957), que consiste em homogeneizar a amostra com clorofórmio:metanol (2:1), adicionar água destilada, separar as fases imiscíveis, repetir o procedimento, reservar a fase orgânica e combinar as fases aquosas, adicionando a estas clorofórmio:metanol (2:1), separar as fases imiscíveis, descartar as fases aquosas e combinar as fases orgânicas, filtrá-las em $\mathrm{Na}_{2} \mathrm{SO}_{4}$, secar sob fluxo de nitrogênio e ressuspender a amostra em $3 \mathrm{~mL}$ de fase móvel (hexano:isopropanol - 97:3 v/v).
A etapa de injeção em cromatógrafo líquido de alta eficiência foi realizada no Laboratório de Bromatologia do Departamento de Nutrição da Faculdade de Saúde Pública da Universidade de São Paulo, de acordo com Vicente (2003). A identificação e quantificação dos compostos colesterol e óxidos foram realizadas com detector UV/visível com varredura da empresa Shimadzu (modelo 10A VP), sendo selecionados os comprimentos de onda de absorção máxima $206 \mathrm{~nm}$ para colesterol e óxidos $7 \alpha-\mathrm{OH}$, $7 \beta-\mathrm{OH}$ e $25-\mathrm{OH}$ e $233 \mathrm{~nm}$ para o óxido 7-Ceto. Os padrões dos OsC foram obtidos da empresa Steraloids (Wilton, EUA). Foi realizada corrida isocrática de $25 \mathrm{~min}$, com fluxo de $1,0 \mathrm{~mL} / \mathrm{min}$. A fase móvel foi filtrada em membrana de $0,45 \mu \mathrm{m}$ e degaseificada. Para a separação dos compostos foi utilizada a coluna de fase intermediária Luna $\mathrm{CN}$ $5 \mu$ da marca Phenomenex, com $25 \mathrm{~cm}$ de comprimento. Foram injetados $20 \mu \mathrm{L}$ de amostra e de cinco diferentes concentrações de padrão de colesterol (de $0,15 \mathrm{mg} / \mathrm{mL}$ a $1,89 \mathrm{mg} / \mathrm{mL})$ e de cada óxido $(4,6 \mu \mathrm{g} / \mathrm{mL}$ a $230,0 \mu \mathrm{g} / \mathrm{mL})$ analisado.

Os limites de detecção e quantificação foram determinados segundo Leite (1998), utilizando-se soluções padrões (Tabela I). Os valores de repetibilidade foram obtidos através de múltiplas injeções $(n=3)$ do ponto mais diluído da curva-padrão. Os experimentos de recuperação foram realizados de acordo com Vicente e Torres (2007), obtendo-se os valores apresentados na Tabela I.

TABELA I - Limites de detecção e quantificação $(n=7)$ para colesterol $(\mu \mathrm{g})$ e óxidos de colesterol $(\mu \mathrm{g}), \%$ de recuperação $(n=6)$ de métodos de extração e quantificação e \% dos valores de repetibilidade

\begin{tabular}{lcccc}
\hline Composto & $\begin{array}{c}\text { Limite de } \\
\text { Detecção }\end{array}$ & $\begin{array}{c}\text { Limite de } \\
\text { Quantificação }\end{array}$ & $\begin{array}{c}\text { Recuperação } \\
(\%)\end{array}$ & $\begin{array}{c}\text { Repetição } \\
(\%)\end{array}$ \\
\hline Colesterol & $8,52.10^{-4}$ & $2,84.10^{-3}$ & $92,8 \pm 2,0$ & 2,76 \\
7-Ceto & 0,01 & 0,02 & $94,4 \pm 1,7$ & 1,69 \\
$25-\mathrm{OH}$ & 0,12 & 0,41 & $91,2 \pm 1,8$ & 1,27 \\
$7 \alpha-\mathrm{OH}$ & 0,10 & 0,33 & $91,3 \pm 2,2$ & 2,39 \\
$7 \beta-\mathrm{OH}$ & 0,03 & 0,09 & $91,9 \pm 2,5$ & 0,71 \\
\hline
\end{tabular}

\section{Planejamento e análise estatística}

O delineamento experimental foi o inteiramente casualizado. Após a observação das pressuposições da Análise de Variância (ANOVA) paramétrica, optou-se em avaliar a existência ou não de diferenças estatísticas entre os tratamentos através da ANOVA não-paramétrica, utilizando-se o teste de Mann-Whitney $(\mathrm{p}<0,05)$. 


\section{RESULTADOS E DISCUSSÃO}

\section{Composição centesimal/ Valor calórico/ Cloretos}

Os valores da composição centesimal, calorias e cloretos do peixe mandim in natura e beneficiado encontram-se na Tabela II. Para eliminar a influência da umidade, foram calculados também os respectivos teores em base seca.

Na mesma coluna, médias seguidas por letras diferentes diferem significativamente $(p<0,05)$.

O mandim beneficiado apresentou teor de umidade de $40,31 \mathrm{~g} / 100 \mathrm{~g}$, inferior aos 70,13 g/100g encontrados no in natura; redução esperada, uma vez que a salga baseia-se no princípio da desidratação osmótica, onde os tecidos do peixe atuam como membranas permeáveis, permitindo a entrada do sal por difusão à medida que ocorre sua desidratação (Burgess et al., 1967), e que o processo de secagem corresponde à redução de umidade (Gioielli, Pitombo, 1998). Mas, de acordo com o Regulamento da Inspeção Industrial e Sanitária de Produtos de Origem Animal (R.I.I.S.P.O.A.) (Brasil, 1997), esse nível de umidade do peixe beneficiado está acima do recomendado: o pescado salgado-seco não deve conter mais que $35 \mathrm{~g} / 100 \mathrm{~g}$ de água. Em estudo com camarão salgado e seco, Kraemer (2000) e Sampaio (2004) também encontraram valores de umidade superiores ao permitido pela legislação brasileira, $51,04 \mathrm{~g} / 100 \mathrm{~g}$ e $40,40 \mathrm{~g} / 100 \mathrm{~g}$ a 55,50 g/100 g, respectivamente, associados a condições inadequadas de secagem e/ ou armazenamento. Como são escassos na literatura dados sobre a composição química de peixes beneficiados de forma semelhante ao mandim, foram feitas algumas comparações com outro tipo de pescado.

Já Szenttamásy et al. (1993), em análise do peixe pacu (Piaractus mesopotamicus), proveniente de reservatórios de piscicultura de Piracicaba-SP, detectaram teores de umidade de 75,54 g/100 g nas amostras in natura e $11,36 \mathrm{~g} / 100 \mathrm{~g}$ nas salgado-secas, devendo-se considerar, no entanto, que os processos de salga e secagem foram realizados em laboratório, sob condições padronizadas.

O mandim, enquanto in natura, pode ser classificado dentro da faixa de umidade referida para pescado. $\mathrm{Na}$ composição química desses produtos, a água representa o principal componente, na proporção de $64 \mathrm{~g} / 100 \mathrm{~g}$ a $90 \mathrm{~g} / 100 \mathrm{~g}$ (Badolato et al., 1994). Estudando a composição centesimal de peixes do mesmo habitat do mandim (Lagoa Mundaú-AL), Menezes (2006) referiu para filés frescos de tainha (Mugil cephalus) e camurim (Centropomus undecimalis) níveis de umidade de 78,40 g/100 g e $79,62 \mathrm{~g} / 100 \mathrm{~g}$, respectivamente, coerentes com os valores encontrados no presente estudo.

Em geral, no início do processo de salga ocorre redução de umidade, mas aumento do teor de cinzas, devido ao sal (Ogawa et al., 1999). No mandim, foram encontrados teores de cinzas de 4,84 g/100 g no in natura e 23,18 g/100 g no beneficiado. No que se refere a esse parâmetro, o peixe beneficiado está em conformidade com a legislação brasileira, que estabelece que o pescado salgado-seco deve apresentar resíduo mineral fixo menor que 25,00 g/100 g (Brasil, 1997). Szenttamásy et al. (1993) detectaram no pacu valores menores, $1,82 \mathrm{~g} / 100 \mathrm{~g}$ no in natura e $17,76 \mathrm{~g} / 100 \mathrm{~g}$ no salgado-seco, semelhante a Natarajan e Sreenivasan (1961), que encontraram em peixes de água doce frescos variação do teor de cinzas de $0,81 \mathrm{~g} / 100 \mathrm{~g}$ a $1,95 \mathrm{~g} / 100 \mathrm{~g}$, referindo que quando o peixe é analisado inteiro, essa concentração pode chegar até 5,14 g/100 g. O mandim foi analisado apenas sem as vísceras, incluindo a cabeça, pois essa é a forma de consumo usual na região. Menezes (2006) também detectou nos filés frescos da tainha e do camurim teores menores, $1,06 \mathrm{~g} / 100 \mathrm{~g}$ e $1,09 \mathrm{~g} / 100 \mathrm{~g}$, respectivamente.

Já os percentuais protéicos encontrados por Menezes (2006) nos filés frescos da tainha, $20,85 \mathrm{~g} / 100 \mathrm{~g}$, e do camurim, $18,29 \mathrm{~g} / 100 \mathrm{~g}$, foram maiores que os $15,30 \mathrm{~g} / 100 \mathrm{~g}$ detectados no mandim in natura. Em peixes de água doce, autores também referiram valores um pouco maiores: Maia et al. (1999) detectaram 18,60 g/100 g no curimatã comum

TABELA II - Composição centesimal, valor calórico e teor de cloretos do peixe mandim (Arius spixii) in natura e beneficiado

\begin{tabular}{lccccccc}
\hline \multirow{2}{*}{ Peixe Mandim } & \multicolumn{7}{c}{ Composição Centesimal (g/100g) } \\
& Umidade & Cinzas & Proteínas & Lipídios & Carboidratos & $\begin{array}{c}\text { Calorias } \\
(\mathrm{kcal} / 100 \mathrm{~g})\end{array}$ & $\begin{array}{c}\text { Cloretos } \\
(\mathrm{g} / 100 \mathrm{~g})\end{array}$ \\
\hline In natura (Base Úmida*) & $70,13^{\mathrm{a}}$ & 4,84 & 15,30 & 8,67 & 1,39 & 145,00 & 0,85 \\
In natura (Base Seca) & - & $16,42^{\mathrm{a}}$ & $51,73^{\mathrm{a}}$ & $28,89^{\mathrm{a}}$ & $4,67^{\mathrm{a}}$ & $486,00^{\mathrm{a}}$ & $2,92^{\mathrm{a}}$ \\
Beneficiado (Base Úmida*) & $40,31^{\mathrm{b}}$ & 23,18 & 22,63 & 13,58 & 1,40 & 218,00 & 17,02 \\
Beneficiado (Base Seca) & - & $38,84^{\mathrm{b}}$ & $38,07^{\mathrm{b}}$ & $22,85^{\mathrm{a}}$ & $2,24^{\mathrm{b}}$ & $367,00^{\mathrm{b}}$ & $57,65^{\mathrm{b}}$ \\
\hline
\end{tabular}

*Média de 15 amostras analisadas em duplicata. 
(Prochilodus cearensis), Siqueira (2001) encontrou em tilápia (Oreochromis miloticus) 18,20 g/100 g e Szenttamásy et al. (1993) detectaram 19,80 g/100 g no pacu in natura.

No mandim beneficiado, o teor de proteínas foi de $22,63 \mathrm{~g} / 100 \mathrm{~g}$. Valor maior foi detectado por Szenttamásy et al. (1993) no pacu salgado-seco, 52,53 g/100 g (base úmida). Quando comparados em base seca, os percentuais protéicos do mandim, $51,73 \mathrm{~g} / 100 \mathrm{~g}$ no in natura e $38,07 \mathrm{~g} / 100 \mathrm{~g}$ no beneficiado, mostram que após o beneficiamento houve redução do teor de proteínas.

Na secagem do pescado salgado, podem ocorrer dois tipos de reações relevantes, que implicam em perdas do valor nutricional: destruição parcial dos nutrientes pela exposição à alta temperatura, e interação de compostos produzidos durante o processamento (Labuza, 1973).

Os dados da Tabela II demonstram que o único parâmetro da composição centesimal para o qual não houve diferença significativa entre as formas estudadas $(\mathrm{p} \geq 0,05)$ foi lipídios. Os teores de lipídios detectados foram de $8,67 \mathrm{~g} / 100 \mathrm{~g}$ no mandim in natura e 13,58 g/100 g no beneficiado. No pacu in natura e salgado-seco, Szenttamásy et al. (1993) encontraram valores de 3,79 g/100 g e $18,31 \mathrm{~g} / 100 \mathrm{~g}$, respectivamente. A salga, apesar de não ter contribuído significativamente para a redução no percentual lipídico, pode favorecer alterações oxidativas, dependendo de fatores como temperatura, intensidade e composição da luz, tempo de ação do sal e tensão parcial do oxigênio (Effemberg, Schotte, 1972).

Para peixes in natura, teor menor de lipídeos também foi apontado por Menezes (2006), que detectou 2,50 g/100g nos filés da tainha e do camurim. Enumerando 56 espécies de peixes de água doce, Henderson e Tocher (1987) constataram que os filés frescos apresentaram teores maiores, de $0,70 \mathrm{~g} / 100 \mathrm{~g}$ a $25,80 \mathrm{~g} / 100 \mathrm{~g}$. Variação também maior de lipídios para peixes frescos foi relatada por Natarajan e Sreenivasan (1961), de 0,17 g/100g a $10,10 \mathrm{~g} / 100 \mathrm{~g}$. Kinsella et al. (1977) enfatizaram que a variação em teores lipídicos de pescado é influenciada pela idade do animal, fonte de captura, sazonalidade e métodos de análise.

Quanto aos carboidratos, os teores de 1,39 g/100g e $1,40 \mathrm{~g} / 100 \mathrm{~g}$ encontrados no mandim in natura e beneficiado, respectivamente, foram superiores a dados obtidos na literatura. Luzia (2000) relatou que níveis de carboidratos baixos ou inexistentes são esperados em pescado. O teor é geralmente inferior a $1,00 \mathrm{~g} / 100 \mathrm{~g}$. Da mesma forma, Ogawa e Maia (1999) descreveram que o conteúdo de carboidratos em peixes é de $0,30 \mathrm{~g} / 100 \mathrm{~g}$ a $1,00 \mathrm{~g} / 100 \mathrm{~g}$, mas que alguns pescados podem estocar parte de sua reserva energética como glicogênio, o qual contribui para o aumento do teor. No camarão salgado-seco, Sampaio
(2004) encontrou, em base úmida, 2,30 g/100g de carboidratos. A diminuição significativa de carboidratos no mandim após o beneficiamento, demonstrada pelos valores, em base seca, de 4,67 g/100g no in natura e 2,24 g/100g no beneficiado, foi determinada pela adição do sal.

As alterações, na composição centesimal do mandim beneficiado, levaram a perda de cerca de $25 \%$ de seu valor calórico. Em base seca, foram detectadas $367 \mathrm{kcal} / 100 \mathrm{~g}$, inferiores as $486 \mathrm{kcal} / 100 \mathrm{~g}$ do in natura. Em base úmida, níveis calóricos menores que os $145 \mathrm{kcal} / 100 \mathrm{~g}$ do mandim in natura e os $218 \mathrm{kcal} / 100 \mathrm{~g}$ do beneficiado foram apontados por Menezes (2006) para os filés frescos da tainha e do camurim, 105,91 kcal/100g e 95,66 kcal/100g, respectivamente, e por Sampaio (2006) para o camarão salgado-seco, de $107,72 \mathrm{kcal} / 100 \mathrm{~g}$ a $110,72 \mathrm{kcal} / 100 \mathrm{~g}$. Como o valor calórico está correlacionado ao teor de lipídios (Luzia, 2000), essa diferença entre os resultados está dentro do esperado. Para o camarão salgado-seco, Sampaio (2004) encontrou conteúdo lipídico de apenas 1,12 g/100g.

Ainda na Tabela II, o teor de 17,02 g/100g de cloretos do mandim beneficiado, maior em relação a $0,85 \mathrm{~g} / 100 \mathrm{~g}$ do in natura, foi resultado da incorporação do sal no processo de salga. A carne dos peixes tem sal em torno de $0,08 \mathrm{~g} / 100 \mathrm{~g}$ a $1,00 \mathrm{~g} / 100 \mathrm{~g}$, mas eleva-se artificialmente esse conteúdo através da aplicação de cloreto de sódio (Grecchi, 1972). A concentração de cloretos do mandim beneficiado é considerada satisfatória. De acordo com o R.I.I.S.P.O.A. (Brasil, 1997), o teor de cloretos do pescado salgado deve estar compreendido entre $12,00 \mathrm{~g} / 100 \mathrm{~g}$ e $18,00 \mathrm{~g} / 100 \mathrm{~g}$. Resultado semelhante foi relatado por Szenttamásy et al. (1993) para o pacu salgado-seco, $17 \mathrm{~g} / 100 \mathrm{~g}$ de cloretos.

\section{Perfil de ácidos graxos}

No peixe mandim in natura e beneficiado, os ácidos graxos de maior predominância, dentre os 22 detectados (Tabela III), foram, respectivamente: palmítico (C16:0), $41,19 \%$ e $39,80 \%$; oléico (C18:1n9), 16,72\% e 17,60\%; esteárico (C18:0), 10,40\% e 9,43\%; e palmitoléico (C16:1n 7$), 7,09 \%$ e $7,70 \%$.

Dentre os ácidos graxos saturados predominantes no mandim, o palmítico é um dos mais hipercolesterolêmicos e aterogênicos, sendo considerado fator dietético de risco para o desenvolvimento de doença cardiovascular (Curi et al., 2002). Menezes (2006), nos filés frescos da tainha e do camurim, encontrou valores menores desse ácido, 12,97 e $14,38 \%$, respectivamente. Mas em peixes de água doce, Castelo, Amaya e Strong (1980), Maia, Rodriguez-Amaya e Amaya-Farfán (1983) e Pezzato (1990) detectaram variação maior, 34,00 a 49,00\%. 
TABELA III - Ácidos graxos (\% de área) do peixe mandim (Arius spixii) in natura e beneficiado

\begin{tabular}{|c|c|c|}
\hline \multirow[t]{2}{*}{ Ácidos Graxos } & \multicolumn{2}{|c|}{ Peixe Mandim } \\
\hline & In natura* & Beneficiado* \\
\hline Capróico (C6:0) & $0,38^{\mathrm{a}}$ & $1,12^{\mathrm{a}}$ \\
\hline Mirístico (C14:0) & $2,30^{\mathrm{a}}$ & $2,25^{\mathrm{a}}$ \\
\hline Pentadecanóico (C15:0) & $1,04^{\mathrm{a}}$ & $0,48^{\mathrm{a}}$ \\
\hline Palmítico (C16:0) & $41,19^{\mathrm{a}}$ & $39,80^{\mathrm{a}}$ \\
\hline Palmitoléico (C16:1n7) & $7,09^{\mathrm{a}}$ & $7,70^{\mathrm{a}}$ \\
\hline Heptadecanóico (C17:0) & $1,19^{\mathrm{a}}$ & $1,40^{\mathrm{b}}$ \\
\hline cis-10-heptadecenóico (C17:1) & $0,74^{\mathrm{a}}$ & $0,40^{\mathrm{a}}$ \\
\hline Esteárico $(\mathrm{C} 18: 0)$ & $10,40^{\mathrm{a}}$ & $9,43^{\mathrm{a}}$ \\
\hline Oléico (C18:1n9) & $16,72^{\mathrm{a}}$ & $17,60^{\mathrm{a}}$ \\
\hline Elaídico(C18:1n9t) & $1,10^{\mathrm{a}}$ & $1,70^{\mathrm{a}}$ \\
\hline Linoléico (C18:2n6) & $2,55^{\mathrm{a}}$ & $1,62^{\mathrm{a}}$ \\
\hline$\gamma$-linolênico (C18:3n6) & $0,59^{\mathrm{a}}$ & $0,29^{\mathrm{a}}$ \\
\hline$\alpha$-linolênico (C18:3n3) & $2,09^{\mathrm{a}}$ & $1,77^{\mathrm{a}}$ \\
\hline Eicosenóico (C20:1n9) & $0,56^{\mathrm{a}}$ & $0,19^{b}$ \\
\hline Eicosadienóico (C20:2n6) & $0,53^{\mathrm{a}}$ & $0,00^{\mathrm{b}}$ \\
\hline Eicosatrienóico (C20:3n3) & $0,98^{\mathrm{a}}$ & $0,67^{\mathrm{a}}$ \\
\hline Eicosapentaenóico (C20:5n3) & $1,57^{\mathrm{a}}$ & $1,88^{\mathrm{a}}$ \\
\hline Docosadienóico (C22:2n6) & $2,36^{\mathrm{a}}$ & $7,07^{\mathrm{b}}$ \\
\hline Docosahexaenóico (C22:6n3) & $3,87^{\mathrm{a}}$ & $2,19^{\mathrm{a}}$ \\
\hline Tricosanóico (C23:0) & $0,80^{\mathrm{a}}$ & $1,49^{\mathrm{a}}$ \\
\hline Lignocérico (C24:0) & $1,81^{\mathrm{a}}$ & $0,97^{\mathrm{a}}$ \\
\hline Nervônico (C24:1) & $0,13^{\mathrm{a}}$ & $0,10^{\mathrm{a}}$ \\
\hline$\Sigma$ Saturados & $59,11^{\mathrm{a}}$ & $56,94^{\mathrm{a}}$ \\
\hline sMonoinsaturados & $26,34^{\mathrm{a}}$ & $27,69^{\mathrm{a}}$ \\
\hline$\Sigma$ Poliinsaturados & $14,54^{\mathrm{a}}$ & $15,49^{\mathrm{a}}$ \\
\hline$\Sigma$ n3 & $8,51^{\mathrm{a}}$ & $6,51^{b}$ \\
\hline$\Sigma \mathrm{n} 6$ & $6,03^{\mathrm{a}}$ & $8,98^{\mathrm{a}}$ \\
\hline Razão n6/n3 & $0,71: 1^{\mathrm{a}}$ & $1,38: 1^{\mathrm{a}}$ \\
\hline $\mathrm{EPA}+\mathrm{DHA}$ & $5,44^{\mathrm{a}}$ & $4,07^{b}$ \\
\hline
\end{tabular}

*Média de 12 amostras analisadas em duplicata. Na mesma linha, médias seguidas por letras diferentes diferem significativamente $(\mathrm{p}<0,05)$.

Já o ácido saturado esteárico, por não ter efeito sobre as lipoproteínas sangüíneas, é considerado neutro (KrisEtherton, Yu, 1997). Maia (1992) encontrou para o peixe de água doce tambaqui (Colossama macropomum) 9,80\% de esteárico. Valores diferentes foram apontados por Menezes (2006), que encontrou nos filés frescos da tainha $4,26 \%$, e do camurim $11,93 \%$.

O ácido mirístico (C14:0), detectado em teores pouco expressivos de $2,30 \%$ no mandim in natura e $2,25 \%$ no beneficiado, é o ácido saturado hipercolesterolêmico e aterogênico de maior potencialidade (Kris-Etherton, Yu, 1997). Nos filés frescos da tainha e do camurim, Menezes
(2006) detectou valores maiores, 7,66\% e 7,09\%, respectivamente.

Em relação aos ácidos graxos monoinsaturados, o oléico, detectado no presente estudo com predominância, tem significativo efeito hipolipidêmico quando substitui gordura saturada na dieta, diminuindo os níveis séricos de colesterol e triacilgliceróis (Mahan, Escott-Stump, 2005), o que engrandece o valor nutricional do mandim. Para o tambaqui, Maia (1992) relatou teor maior desse ácido, 40,10\%, mas no curimbatá (Prochilodus scrofa), peixe de água doce, esse mesmo autor encontrou concentração menor, $15,30 \%$. Teores ainda menores foram detectados por Menezes (2006), 6,27\% nos filés frescos da tainha e 4,88\% nos do camurim.

O ácido palmitoléico, segundo principal ácido monoinsaturado do mandim, também foi encontrado por Maia (1992) no curimbatá e no tambaqui, em concentrações de $16,30 \%$ e $6,30 \%$, respectivamente.

Dentre os ácidos graxos poliinsaturados, formas importantes de ômega-3 e ômega- 6 foram detectadas no mandim in natura e beneficiado, respectivamente: docosahexaenóico (C22:6n3 - DHA), 3,87\% e 2,19\%; $\alpha$-linolênico (C18:3n3), 2,09\% e 1,77\%; eicosapentaenóico (C20:5n3 - EPA), 1,57\% e 1,88\%; e linoléico (C18:2n6), 2,55\% e 1,62\%.

Os ácidos graxos poliinsaturados ômega-3 e ômega6 exercem funções indispensáveis para o organismo humano, e só podem ser adquiridos através da dieta, por esse motivo, são considerados essenciais (Martin et al. 2006). O impacto dos ácidos ômega-3 na doença cardiovascular, artrite, câncer e outras doenças crônicas, e estados imunológicos e mentais alterados está sendo bastante estudado, assim como a associação da deficiência de ômega-6 com importantes implicações clínicas, inclusive retardo mental, lesões de pele e insuficiência reprodutiva (Mahan, Escott-Stump, 2005).

Menezes (2006) encontrou os seguintes teores de ácidos poliinsaturados: linoléico $6,28 \%, \alpha$-linolênico $5,61 \%$, EPA 5,42\%, e DHA 5,05\%, nos filés frescos da tainha; EPA 4,28\%, linoléico 4,08\%, $\alpha$-linolênico 2,20\% e DHA $0,93 \%$, nos do camurim.

A comparação do mandim beneficiado com outros peixes salgado-secos ficou prejudicada pela escassez de trabalhos na literatura que identifiquem e quantifiquem completamente os seus ácidos graxos.

No mandim beneficiado, houve aumento do teor do ácido saturado heptadecanóico (C17:0) de 1,19\%, in natura, para $1,40 \%$, e do ácido poliinsaturado docosadienóico (C22:2n6) de 2,36\%, in natura, para $7,07 \%$, porém, em relação aos ácidos monoinsaturado eicosenóico (C20:1n9) e poliinsaturado eicosadienóico 
(C20:2n6), respectivamente, houve diminuição de 0,56\%, in natura, para $0,19 \%$ e de $0,53 \%$, in natura, para teor 0 . Esses foram os únicos ácidos graxos para os quais, isoladamente, houve diferença significativa entre o peixe in natura e o beneficiado $(\mathrm{p}<0,05)$.

$\mathrm{Na}$ Tabela III, são também apresentados para o mandim in natura e beneficiado, respectivamente, os percentuais totais dos ácidos graxos: saturados, $59,11 \%$ e $56,94 \%$; monoinsaturados, $26,34 \%$ e $27,69 \%$; poliinsaturados, $14,54 \%$ e $15,49 \%$; EPA + DHA, 5,44\% e 4,07\%. A redução de ômega-3 (EPA + DHA) $(\mathrm{p}<0,05)$ no mandim beneficiado demonstra alteração no perfil de ácidos graxos. Menezes (2006), nos filés frescos da tainha e do camurim, respectivamente, encontrou teores de $49,77 \%$ e $65,71 \%$ de saturados, $20,01 \%$ e $17,45 \%$ de monoinsaturados, $30,12 \%$ e $17,88 \%$ de poliinsaturados, e $10,47 \%$ e $5,21 \%$ de EPA + DHA.

O EPA é o ácido graxo mais importante da dieta, por ser precursor dos eicosanóides (Chen et al., 1995). E o DHA, segundo Childs, King e Knopp (1990), diminui a concentração sangüínea do LDL-colesterol. Levando em consideração esses e outros benefícios para a saúde, a determinação nesse trabalho da soma desses dois ácidos visou a melhor avaliação nutricional da espécie estudada.

Com o mesmo objetivo, foi determinada a proporção entre os ácidos ômega-6 e ômega-3 (Tabela III), 0,71:1 no mandim in natura e 1,38:1 no beneficiado, não sendo constatada em relação a esse parâmetro diferença significativa entre as formas analisadas $(\geq 0,05)$. A Japan Society of Lipid Nutrition recomenda que a razão $\mathrm{n} 6 / \mathrm{n} 3$ seja de no máximo 4:1 para adultos saudáveis e de 2:1 na prevenção de doenças crônicas em idosos (Uauy, Mena, Valenzuela, 1999). Menezes (2006) apontou para os filés frescos da tainha e do camurim razão n6/n3 de 1:2.

Os seres humanos podem dessaturar e alongar o ácido $\alpha$-linolênico em EPA e DHA, porém o excesso de ômega- 6 na dieta, acima da proporção ótima n6/n3 de 2:1 a 3:1, satura as enzimas e impede essa conversão (KrisEtherton, 2000 apud Mahan, Escott-Stump, 2005).

\section{Colesterol e óxidos de colesterol $(\mathrm{OsC})$}

Na Tabela IV, encontram-se os resultados obtidos para o colesterol e OsC no peixe mandim in natura e beneficiado.

Os teores de colesterol do mandim foram de $82,66 \mathrm{mg} / 100 \mathrm{~g}$ no in natura e $61,30 \mathrm{mg} / 100 \mathrm{~g}$ no beneficiado. Essa diferença, estatisticamente significativa $(\mathrm{p}<0,05)$, demonstra que o beneficiamento favoreceu a redução do conteúdo total de colesterol.

Valores de colesterol entre $50 \mathrm{mg} / 100 \mathrm{~g}$ e $90 \mathrm{mg} / 100 \mathrm{~g}$ foram encontrados por Kinsella et al. (1977)
TABELA IV - Teor de colesterol e óxido de colesterol (7-Ceto) do peixe mandim (Arius spixii) in natura e beneficiado

\begin{tabular}{lcc}
\hline Peixe Mandim & Colesterol $(\mathrm{mg} / 100 \mathrm{~g})$ & 7-Ceto $(\mu \mathrm{g} / \mathrm{g})$ \\
\hline In natura* $^{*}$ & $82,66^{\mathrm{a}}$ & $8,31^{\mathrm{a}}$ \\
Beneficiado* $^{\mathrm{b}}$ & $61,30^{\mathrm{b}}$ & $17,90^{\mathrm{b}}$ \\
\hline
\end{tabular}

*Média de 15 amostras analisadas em duplicata. Na mesma coluna, médias seguidas por letras diferentes diferem significativamente $(\mathrm{p}<0,05)$.

e Sweeney e Weihrauch (1977) em peixes de água doce. Níveis maiores foram relatados por Menezes (2006) para os filés frescos da tainha, $188,00 \mathrm{mg} / 100 \mathrm{~g}$, e do camurim, $187,52 \mathrm{mg} / 100 \mathrm{~g}$. Segundo Bragagnolo (1997), essa variação entre os resultados pode ser atribuída a uma série de fatores, tais como espécie, idade, sexo, alimentação disponível, estação do ano, condições de criação e métodos de análise.

A diminuição da concentração de colesterol no mandim beneficiado está associada à degradação desse esterol a outros compostos, mais especificamente ao 7-Ceto, único OsC detectado no presente estudo. No mandim in natura foram encontrados $8,31 \mu \mathrm{g} / \mathrm{g}$ de 7 -Ceto, no beneficiado esse teor aumentou para $17,90 \mu \mathrm{g} / \mathrm{g}$ $(\mathrm{p}<0,05)$. As Figuras 1, 2 e 3 apresentam os cromatogramas da amostra analisada.

O 7-Ceto é o óxido que tem sido utilizado como indicador da oxidação do colesterol em alimentos, por ser produzido em maior quantidade e nos estágios iniciais do processo oxidativo (Rodriguez-Estrada et al., 1997).

Os dados existentes na literatura referentes a alimentos frescos demonstram que originalmente não deveriam conter OsC (Paniangvait et al., 1995). Mas, de acordo com Moura et al. (2002), nem sempre é isso que se observa. Os poucos estudos que quantificaram $\mathrm{OsC}$ em produtos frescos apresentaram resultados bastante variáveis.

O colesterol é instável sob grande variedade de condições, tais como luz, calor, radiação, presença de radicais livres, oxigênio e metais de transição (Paniangvait et al., 1995), parâmetros que têm sido estudados como fatores influenciadores da sua oxidação (Sampaio, 2004).

Pesquisas têm comprovado que a formação dos $\mathrm{OsC}$ em alimentos é especialmente determinada pela combinação de três fatores: disponibilidade de oxigênio, presença de ácidos graxos poliinsaturados e temperatura elevada (Tai, Chen, Chen, 1999). Os hidroperóxidos que resultam da oxidação dos ácidos graxos poliinsaturados iniciam a oxidação do colesterol e podem aumentá-la sinergicamente (Smith, 1987). 


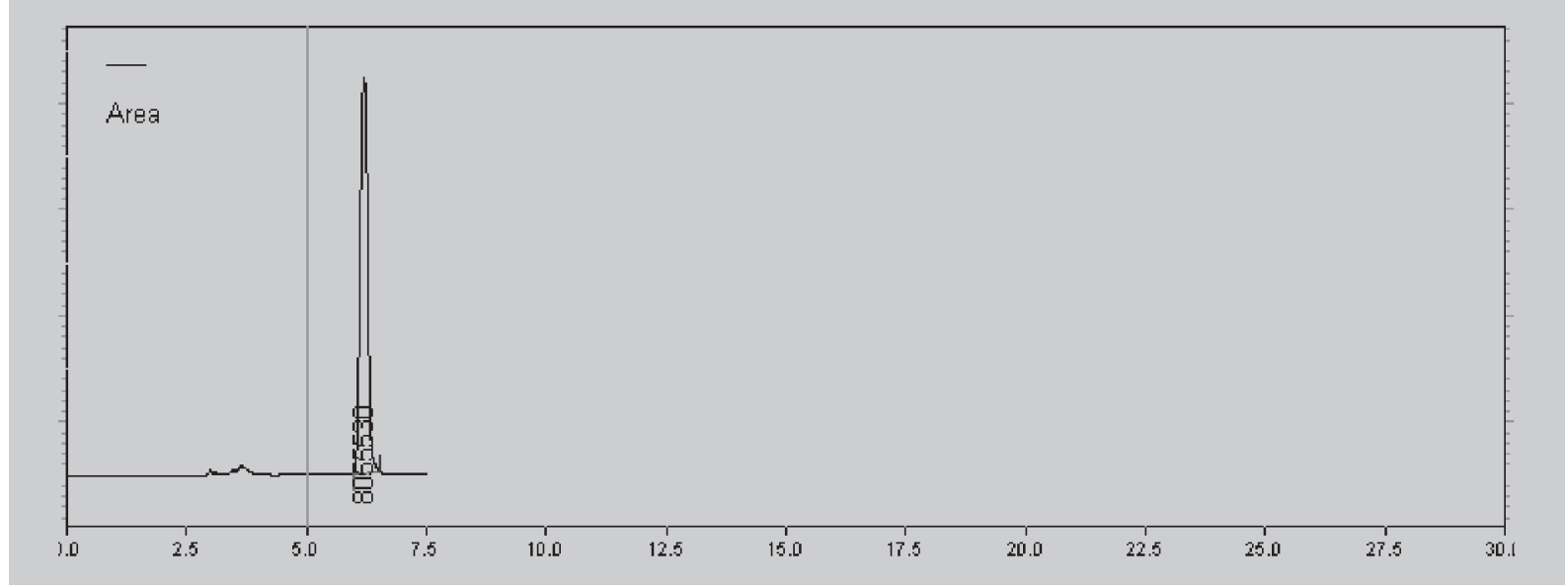

FIGURA 1 - Cromatograma padrão de colesterol a $206 \mathrm{~nm}\left(\right.$ Pico n $\left.^{\circ} 1\right)$

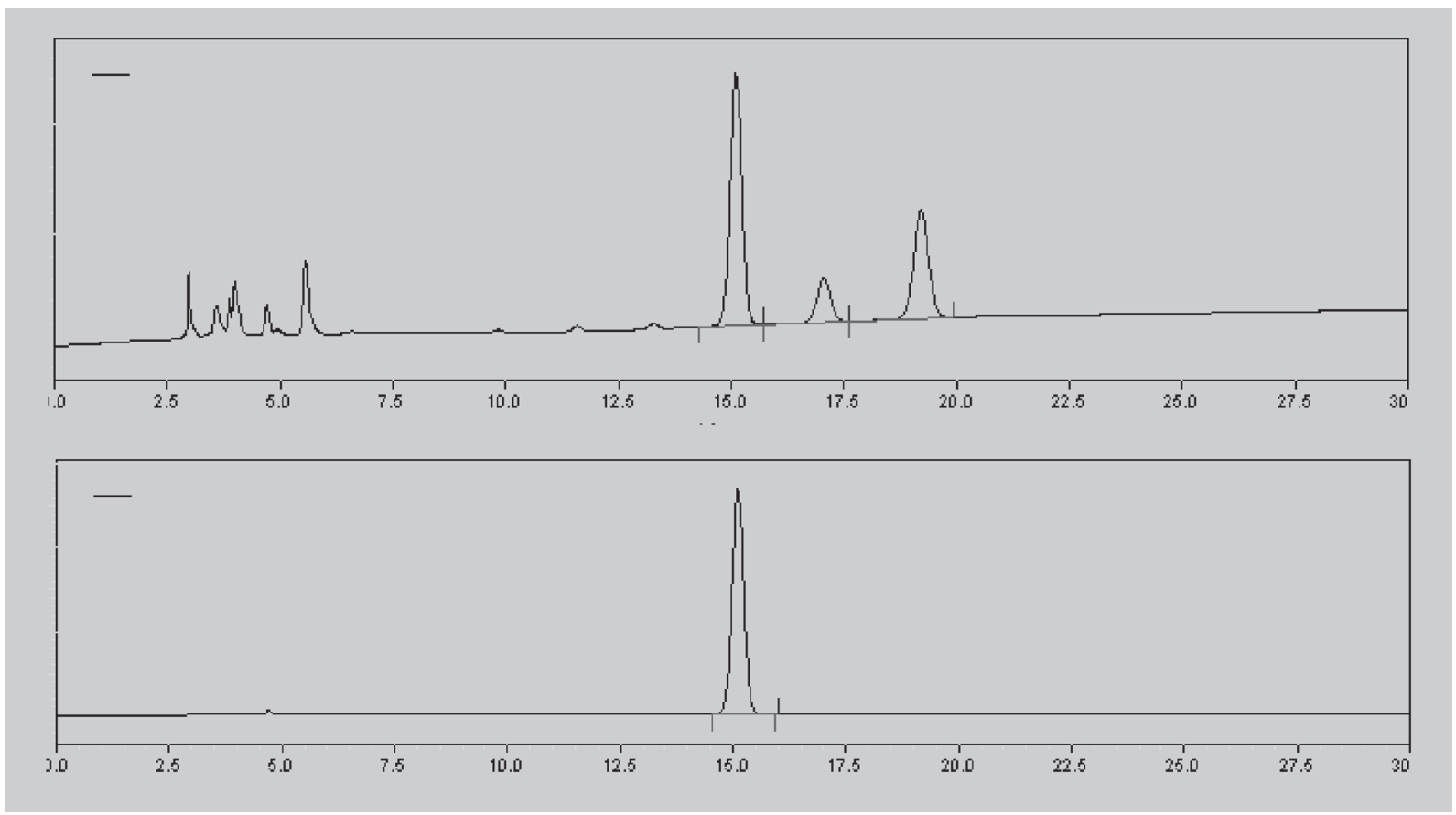

FIGURA 2 - Cromatograma mix de óxidos padrões $\left(1^{\circ}\right.$ cromatograma a $206 \mathrm{~nm}$, os picos referem-se aos seguintes compostos: Pico $2=25-\mathrm{OH}$; Pico $3=7 \alpha-\mathrm{OH}$; Pico $4=7 \beta-\mathrm{OH}$ e $2^{\circ}$ cromatograma a $233 \mathrm{~nm}$, o pico mostrado refere-se ao composto 7-Ceto)

O pescado normalmente está sujeito a procedimentos tecnológicos que envolvem exposição a altas temperaturas (Sebedio et al., 1993). A forma de exposição ao calor se constitui em fator fundamental na oxidação do colesterol, sendo maior a formação dos OsC em alimentos submetidos a aquecimento direto (Morgan, Armstrong, 1992).

Chen e Yeh (1994), estudando a presença de OsC em peixes pequenos secos ao sol, encontraram $7 \alpha-\mathrm{OH}$,

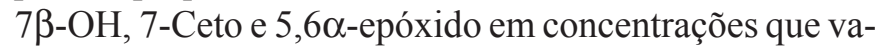
riaram de 4,82 a 65,70 ppm, sendo o $7 \alpha-\mathrm{OH}$ o óxido predominante. Os autores referiram que as condições abusivas de processamento e armazenamento foram o fator princi- pal para a formação desses óxidos.

O mandim, como já descrito, é beneficiado sem obediência a nenhum critério, exposto ao ar, umidade relativa não controlada, e seco com direta incidência da luz do sol por tempo indeterminado.

Sampaio (2006), no camarão salgado-seco, detectou $7 \alpha-\mathrm{OH}, 7 \beta-\mathrm{OH}$ (predominante), 7 -Ceto e $25-\mathrm{OH}$ em teores de 4,52 a 77,30 $\mu \mathrm{g} / \mathrm{g}$, também associando a sua formação a abusos durante a secagem ao sol e/ou armazenamento.

No pescado salgado-seco, o sal também se constitui em facilitador da oxidação lipídica. Associado ao calor, ele torna-se forte pró-oxidante das gorduras, através da ativa- 


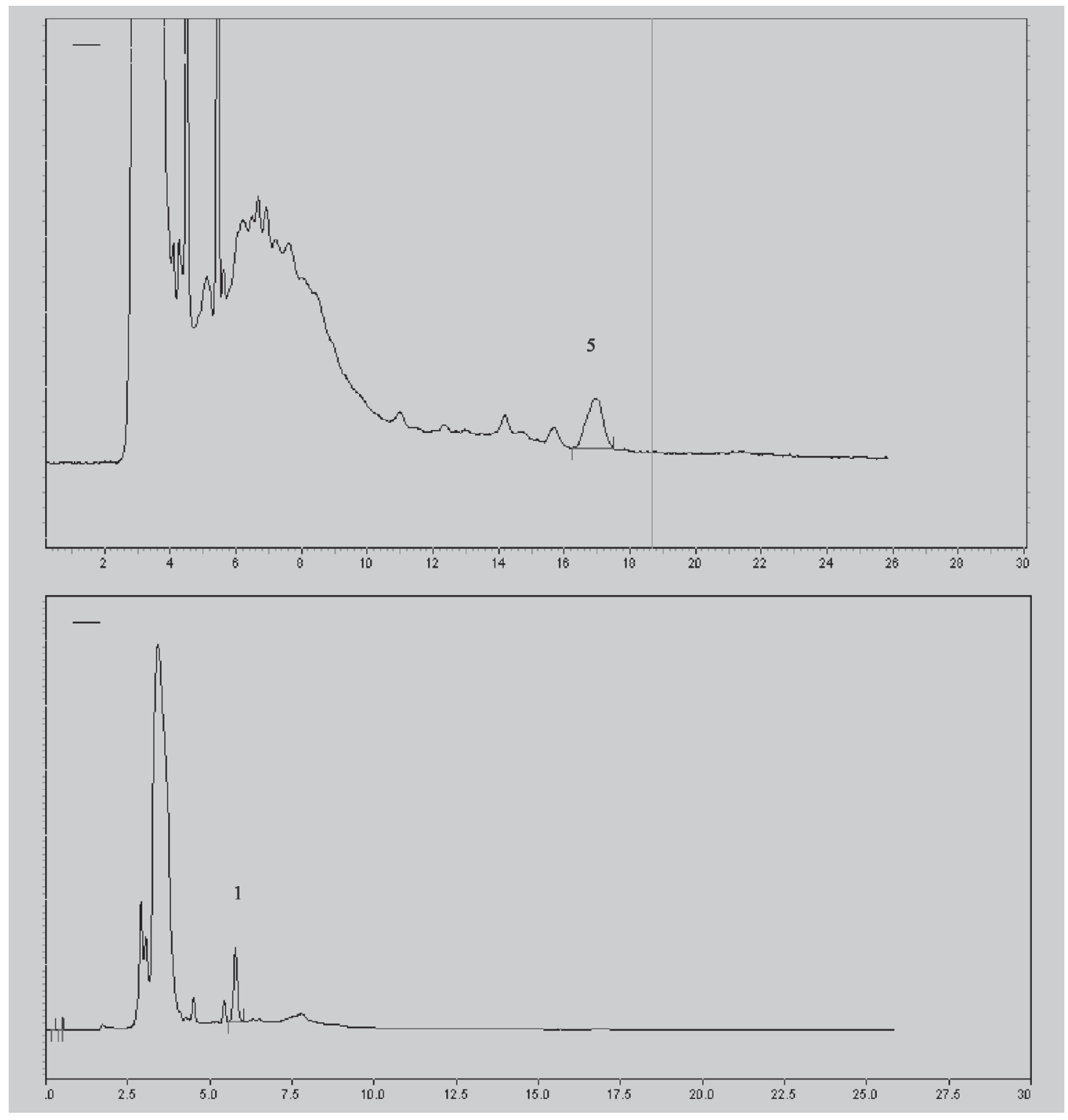

FIGURA 3 - Cromatograma mix de uma amostra de mandim obtido por CLAE ( $1^{\circ}$ cromatograma a $233 \mathrm{~nm}$, para a detecção de 7-Ceto, e $2^{\circ}$ cromatograma a $206 \mathrm{~nm}$, para a detecção de colesterol e outros óxidos)

ção da lipoxidase no músculo (Pardi et al., 2001).

O perfil dos OsC formados em alimentos e as quantidades correspondentes são definidos pelas características desse alimento e interações entre seus componentes e produtos de decomposição, durante o processamento (Morales-Aizpurúa, Tenuta-Filho, 2002).

\section{CONCLUSÕES}

O beneficiamento favoreceu alterações no valor nutricional do peixe mandim (Arius spixii). Observou-se redução do teor de proteínas, carboidratos, calorias e ácidos graxos poliinsaturados ômega-3, além de evolução do processo de oxidação do colesterol, constatada pelo aumento da concentração do óxido 7-cetocolesterol (7-Ceto), que se iniciou no produto fresco.

\section{ABSTRACT \\ Effect of processing on nutritional value of the mandim fish (Arius spixii)}

In an attempt to analyze how processing enhances the nutritional value of the mandim fish (Arius spixii) marketed in Maceió-AL, Brazil, the following nutritional components were determined in fresh and processed (salted-dried) fish: 
centesimal composition, calorie count, chloride, fatty acid and cholesterol profile. The presence of cholesterol oxides was also investigated. Respective results for fresh and processed mandim fish were: moisture $(70.13 \%$ and $40.31 \%)$, proteins $(51.73 \%$ and $38.07 \%$, dried), carbohyrdrates $(4.67 \%$ and $2.24 \%$, dried), calories (486 $\mathrm{kcal} / 100 \mathrm{~g}$ and $367 \mathrm{kcal} / 100 \mathrm{~g}$, dried), fatty acids (polyunsaturared $14,54 \%$ and $15,49 \%$, omega-3 8,51\% and $6,51 \%)$, cholesterol $(82.66 \mathrm{mg} / 100 \mathrm{~g}$ and $61.30 \mathrm{mg} / 100 \mathrm{~g})$ and oxides (7-ketocholesterol $8.31 \mu \mathrm{g} / \mathrm{g}$ and $17.90 \mu \mathrm{g} / \mathrm{g}$ ). These figures clearly showed that processing led to significant change in the nutritional value of the mandim fish.

UNITERMS: Fish/ nutritional value. Fish/ processing. Fish/centesimal composition, Chloride/ determination in fish. Fatty acids/determination in fish. Cholesterol oxides/ determination in fish.

\section{AGRADECIMENTOS}

À Fundação de Amparo à Pesquisa do Estado de Alagoas (FAPEAL) e ao PIBIC/CNPq/UFAL, pelas Bolsas de Iniciação Científica concedidas às alunas.

\section{REFERÊNCIAS BIBLIOGRÁFICAS}

AOAC - ASSOCIATION OF OFFICIAL ANALYTICAL CHEMISTS. Washington, D. C. Official methods of analysis. 15.ed. Washington, 1990. 109p.

BADOLATO, E. S. G.; CARVALHO, J. B.; AMARAL MELLO, M. R. P.; TAVARES, M.; CAMPOS, N. C.; AUED-PIMENTEL, S.; MORAIS, C. Composição centesimal, de ácidos graxos e valor calórico de cinco espécies de peixes marinhos nas diferentes estações do ano. Rev.Inst. Adolfo Lutz, v.54, n.1, p.27-35, 1994.

BARUFFALDI, R.; OLIVEIRA, M. N. Fundamentos de tecnologia de alimentos. São Paulo: Atheneu, 1998.317p.

BASTOS, J.R. Influência da secagem sobre algumas propriedades físico-químicas do músculo de cação branco (Carcharynus porosus). Arq. Ciênc. Mar, v.17, n.2, p.7778, 1977.

BRAGAGNOLO, N. Fatores que influenciam o nivel de colesterol, lipídios totais e composição de ácidos graxos em camarão e carne. Campinas, 1997. 123p. [Tese de Doutorado - Faculdade de Engenharia de Alimentos Universidade Estadual de Campinas].
BRASIL. Ministério da Agricultura e do Abastecimento. Secretaria de Defesa Agropecuária. Departamento de Inspeção de Produtos de Origem Animal. Regulamento da inspeção industrial e sanitária de produtos de origem animal. Aprovado pelo Decreto n. 30.691, 29 mar. 1952, alterado pelos Decretos n.1255, 25 jun. 1962, 1236, 02 set. 1994, 1812, 08 fev. 1996 e 2244, 04 jun. 1997. Diário Oficial, Brasília, 05 jun. 1997. Seção 1, p.1155-1156.

BRASIL. Leis, Decretos, etc. Portaria n ${ }^{\circ} 33 / 98$ da Secretaria de Vigilância Sanitária do Ministério da Saúde. Adota os valores constantes das tabelas do anexo desta portaria como níveis de IDR (Ingestão Diária Recomendada) para as vitaminas, minerais e proteínas. Diário Oficial, Brasília, 30 mar. 1998. Seção 1, n.60-E, p.5-6.

BURGESS, G. H. O.; CUTTING, C. L.; LOVERN, J. A.; WATERMAN, J.J. Fish handling \& processing. New York: Chemical Publishing Co. Inc., 1967. 390p.

BURR, M. L. Fish and the cardiovascular systems. Progr. Food Nutr. Sci., v.13, n.3-4, p.291-316, 1989.

CASTELO, F. P.; AMAYA, D. R.; STRONG, F. C. Aproveitamento e características da gordura cavitária do tambaqui, Colossama macropomum (CURVIER, 1818). Acta Amazônica, v.10, n.3, p.557-576, 1980.

CHEN, J.; YEH, G. Cholesterol oxidation products in small sun-dried fish. Food Chem., v.50, n.2, p.167-170, 1994.

CHEN, I-C.; CHAPMAN, F. A.; WEI, C-I.; PORTEIR, K.M.; O' KEEFE, S.F. Differentiation of cultured and wild sturgeon (Acipencer oxyrinchus desotoi) based on fatty acid composition. J. Food Sci., v.60, n.3, p.631-635, 1995.

CHILDS, M. T.; KING, I. B.; KNOPP, R. H. Divergent lipoprotein reponses to fish oils with various ratios of eicosapentaenoic and docosahexaenoic acids. Am. J. Clin. Nutr., v.52, n.4, p.632-39, 1990.

CORNEJO, F. E. P.; NOGUEIRA, R. I.; PARK, K. J. Manual para produção de pescado salgado-seco. Rio de Janeiro: EMBRAPA, 1999, v.39, p.1-20.

COSTA, F. Documentário da ictiofauna da região das lagoas Mundaú/Manguaba. Projeto de Levantamento Ecológico Cultural (PLEC) - segunda etapa. Maceió: SEPLAN/ CDCT/ Governo do Estado de Alagoas, 1980. 200p. 
CSALLANY,A. S.; AYAZ, K. L. Quantitative determination of organic solvent soluble lipofresein pigments in tissues. Lipids, v.11, n.11, p.412-417, 1976.

CURI, R.; POMPÉIA, C.; MIYASAKA, K.; PROCÓPIO, J. Entendendo as gorduras - os ácidos graxos. 1.ed. São Paulo: Editora Manole, 2002. 580p.

EFFENBERGER, G.; SCHOTTE, K. Empaquetado de la carne y productos cárnicos. Zaragoza, Editorial Acribia, 1972. $186 \mathrm{p}$

FOLCH, J.; LEES, M.; SLOANNE STANLEY, G. H. A simple method for the isolation and purification of total lipide from animal tissues. J. Biol. Chem., Baltimore, v.226, n.1, p.497-509, 1957.

FURTADO, S. M. B.; SHIMOKOMAKI, S.; ROMANELLI, P. F.; RODRIGUEZ-AMAYA, D. B. Avaliação da qualidade da carne caprina salgada. Hig. Aliment., v.5, n.18, p.34-38, 1991.

FURTADO, S. M. B.; ROMANELLI, P. F.; MORAES, M.A.C.; SHIMOKOMAKI, M. Efeito da castração e salga na qualidade da carne de caprinos. Hig. Aliment., v.6, n.22, p.23-26, 1992.

GIOIELLI, L. A.; PITOMBO, R. N. M. Conservação de alimentos pelo controle da umidade. In: BARUFFALDI, R.; OLIVEIRA, M.N. Fundamentos de tecnologia de alimentos. 1.ed. São Paulo: Atheneu, 1998. 317p.

GRECCHI, D. Salga de peixe. Rev.Nac. Pesca., v.14, n.120, p.10-13, 1972.

HARTMAN, L.; LAGO, R.C.A. Rapid preparation of fatty acid methyl esters from lipids. Lab. Pract., v.22, n.8, p.475-476, 1973.

HENDERSON, R. J.; TOCHER, D. R. The lipid composition and biochemistry of freshwater fish. Progr. Lipid Res., v.26, n.4, p.281-347, 1987.

HEROLD, P. M.; KINSELLA, J. E. Fish oil consumption and decreased risk of cardiovascular disease: a comparison of findings from animal and human feeding trials. Am. J. Clin. Nutr., v.43, n.4, p.566-598, 1986.

INSTITUTO ADOLFO LUTZ. Normas analíticas do Instituto Adolfo Lutz. Métodos químicos e físicos para análise de alimentos. 4.ed. Brasília, 2005. 1018p.
JESUS, R. S.; MÁRSICO, E.T.; SOUZA, G. X. W.; LESSI, E.; SÃO CLEMENTE, S. C. Comparação entre os processos de salga em salmoura e salga mista do curimatã (Prochilodus nigricans) na região Amazônica. Rev.Bras. Cienc. Vet., v.9, n.3, p.143-146, 2002.

KINSELLA, J. E.; SHIMP, J. L.; MAI, J.; WEIHRAUCH, J. Sterol, phospholipid, mineral content and proximate composition of filets of select freshwater fish species. $J$. Food Bioch., v.1, n.2, p.131-140, 1977.

KOTB, A. R.; HADEED, A. F. A.; AL-BAKER, A. A. Omega-3 polyunsaturated fatty acid content of some popular species of Arabian Gulf fish. Food Chem., v.40, n.2, p.185-190, 1991.

KRAEMER, F.B. Análise microbiológica e determinação fisico-química de amostras de camarão salgado-seco comercializadas no Estado do Rio de Janeiro. Niterói, 2000. 79p. [Dissertação de Mestrado. Faculdade de Veterinária. Universidade Federal Fluminense].

KRIS-ETHERTON, P. M; YU, S. Individual fatty acid effects on plasma lipids and lipoproteins: human studies. Am.J. Clin. Nutr., v.65, Suppl.1, p.1628-1644, 1997.

KRIS-ETHERTON, P. M.; TAYLOR, D. S.; YU-POTH, S.; HUTH, P.; MORIARTY, K.; FISHELL, V.; HARGROVE, R. L.; ZHAO, G.; ETHERTON, T. D. Polyunsaturated fatty acids in the foodchain in the United States. Am. J. Clin.Nutr., 71, 179, 2000. apud MAHAN, L.K.; ESCOTT-STUMP, S. Krause: alimentos, nutrição e dietoterapia. 11. ed. São Paulo: ROCA, 2005. 1179p.

LABUZA, T. P. Effects of dehydration and storage. Food Technol., v.27, n.2, p.20-26, 1973.

LEITE, F. Sensibilidade, seletividade e limites de detecção e quantificação. In: LEITE, F. Validação em análises químicas. 3.ed. Campinas: Editora Átomo, 1998. p.67-69.

LUZIA, L. A. Influência da sazonalidade no valor nutricional e perfil lipídico em cinco espécies populares de pescado. São Paulo, 2000. 104p. [Dissertação de Mestrado - Faculdade de Saúde Pública. Departamento de Nutrição - Universidade de São Paulo].

MAHAN, L. K.; ESCOTT-STUMP, S. Krause: alimentos, nutrição e dietoterapia. 11.ed. São Paulo: ROCA, 2005. 1179 p. 
MAIA, E. L.; RODRIGUEZ-AMAYA, D. B.; AMAYAFARFÁN, J. Proximate, fatty acid and amino acid. Composition of the Brazilian freshwater fish Prochilodus scrofa. Food Chem., v.12, n. p.275-286, 1983.

MAIA, E. L. Otimização da metodologia para caracterização de constituintes lipídicos e determinação da composição em ácidos graxos e aminoácidos de peixes de água doce. Campinas, 1992. 242p. [Tese de Doutorado. Faculdade de Engenharia de Alimentos. Universidade Estadual de Campinas].

MAIA, E. L.; OLIVEIRA, C. C. S.; SANTIAGO, A. P.; CUNHA, F. E. A.; HOLANDA, F. C. A.; SOUSA, J. A. Composição química e classes de lipídeos em peixes de água doce curimatã comum (Prochilodus cearensis). Ciênc. Tecnol. Aliment., v.19, n.3, p.433-437, 1999.

MARTIN, C.A.; MATSUSHITA, M.; SOUZA, N.E.; VISENTAINER, J.E.R.; VISENTAINER, J.V. Ácido graxos poliinsaturados ômega-3 e ômega6: Importância e ocorrência em alimentos. Rev. Nutr, v.19, n.6, p.761-770, 2006.

MENEZES, M. E. S. Valor nutricional de espécies de peixe (água salgada e estuário) do Estado de Alagoas. Maceió, 2006. 122p. [Dissertação de Mestrado. Instituto de Química. Centro de Ciências Exatas e Naturais Universidade Federal de Alagoas].

MORAIS, C.; SILVEIRA, N. F. A. Alguns aspectos da estabilidade química e microbiológica do pescado salgado, prensado e seco. Colet. Ital., v.24, n.2, p.129-136,1994.

MORALES-AIZPURÚA, I. C.; TENUTA-FILHO, A. Óxidos de colesterol: ocorrência em alimentos, formação e efeitos biológicos. Rev. Bras. Ciênc. Farm., v.38, n.4, p.431-442, 2002.

MORALES-AIZPURÚA, I.C.; TENUTA-FILHO, A. Colesterol, 7-cetocolesterol e 25-hidroxicolesterol em maionese. Ciênc. Tecnol. Aliment., v.25, n.3, p.495-499, 2005.

MORGAN, J. N.; ARMSTRONG, D. J. Quantification of oxidation products in egg yolk powder spray-dried with direct heating. J. Food Sci., v.57, n.1, p.43-45, 1992.

MOURA, A.F.P.; TORRES, R.P.; MANCINI-FILHO, J.; TENUTA-FILHO, A. Caracterização da fração lipídica de amostras comerciais de camarão-rosa. Arch. Latinoamericanos Nutr., v.52, n.2, p.207-211, 2002.
NATARAJAN, U. M.; SREENIVASAN, A. Proximate and mineral composition of freshwater fishes. Indian Journal of Fisheries, v.8, n.2, p.422-429, 1961.

NORT, Coletânea de informações práticas a indústria pesqueira. Programa de Pesquisa e Desenvolvimento Pesqueiro do Brasil. 1974. Apud REALE, D.G. Aspectos do pescado salgado: tecnologia e microbiologia. Belém, 1997. 55p. [Monografia de Especialização. Faculdade de Ciências Agrárias do Pará. Universidade Federal do Pará].

OGAWA, M.; MAIA, E. Alterações da carne de pescado por processamento e estocagem. In: OGAWA, M.; MAIA, E. Manual de pesca - Ciência e tecnologia do pescado. São Paulo: Livraria Varela, 1999. v.1, cap.13, p.222-249.

OGAWA, M.; NUNES, M. L.; OGAWA, N. B.P.; DINIZ, F.M.; OETTERER, M.; MARTIN, A. M.; ITÓ, L.S.; MAIA, E. Tecnologia do pescado. In: OGAWA, M.; MAIA, E. Manual de pesca - Ciência e tecnologia do pescado. São Paulo: Livraria Varela, 1999. v.1, cap.16, p.291-388.

PANIANGVAIT, P.; KING, A.J.; JONES, A. D.; GERMAN, B.G. A critical review cholesterol oxides in foods of animal origin.J. Food Sci., v.60, n.6, p.1159-1174, 1995.

PARDI, M. C.; SANTOS, I. F.; SOUZA, E. R.; PARDI, H. S. Ciência, higiene e tecnologia da carne. Goiânia: UFG, 2001. 623p.

PEZZATO, L. E. Efeito de diferentes niveis de gordura animal e vegetal sobre o desempenho e deposição de ácidos graxos em pacu (Piaractus mesopotamicus). Jaboticabal, 1990.91p. [Tese de Doutorado. Faculdade de Ciências Agro-Veterinárias. Universidade Estadual Paulista].

RODRIGUEZ-ESTRADA, M. T.; PENAZZI, G.; CABONI, M. F.; BERTACCO, G.; LERCKER, G. Effect of different cooking methods on some lipid and protein components of hamburgers. Meat Sci., v.45, n.3, p.365-375, 1997.

SAMPAIO, G. R. Ocorrência de óxidos de colesterol e análise do perfil lipídico em camarão salgado-seco. São Paulo, 2004. 93p. [Dissertação de Mestrado. Faculdade de Saúde Pública. Departamento de Nutrição Universidade de São Paulo].

SAMPAIO, G. R.; BASTOS, D. H. M.; SOARES, R. A. M.; QUEIROZ, Y. S.; TORRES E.A.F.S. Fatty acids and cholesterol oxidation in salted dried shrimp. Food Chem., v.95, n.2, p.344-351, 2006. 
SARGENT, J. R.; HENDERSON, R. J. Marine (n-3) polyunsaturated fatty acids. In: HAMILTON, R. J. Developments in oils and fats. London: Blackie Academic and Professional, 1995, p.32-65.

SARGENT, J. R. Fish oils and human diet. Br. J. Nutr., v.78, Suppl.1, p.5-13, 1997.

SEBEDIO, J. L.; RATNAYAKE, W. M. N.; ACKMAN, R. G.; PREVOST, J. Stability of polyunsaturated É-3 fatty acids during deep fat frying of Atlantic mackerel (Scomber scombrus L.). Food Res. Int., v.26, n.3, p.163-172, 1993.

SIQUEIRA, A. A. Z. C. Efeito da irradiação e refrigeração na qualidade e no valor nutritivo da tilápia (Oreochromis miloticus). Piracicaba, 2001.137p. [Dissertação de Mestrado. Escola Superior de Agricultura Luiz de Queiroz. Universidade de São Paulo].

SMITH, L. L. Cholesterol autoxidation 1981-1986. Chem. Phys. Lipids, v.44, n.2-4, p.87-125, 1987.

SWEENEY, J. P.; WEIHRAUCH, J. Summary of data for cholesterol in foods and methods for its determination.Crit. Rev. Food Sci. Nutr., v.8, n. p.131160, 1977.
SZENTTAMÁSY, E. R.; BARBOSA, S. M. V. B.; OETTERER, M.; MORENO, I. A. M. Tecnologia do pescado de água doce: aproveitamento do pacu (Piaractus mesopotamicus). Sci. Agric., v.50, n.2, p.303-310, 1993.

TAI, C. Y.; CHEN, Y. C.; CHEN, B. H. Analysis, formation and inhibition of cholesterol oxidation products in food: an overview (Part I). J. Food Drug Anal, v.7, n.4, p.243-257, 1999.

UAUY, R.; MENA, P.; VALENZUELA, A. Essential fatty acids as determinants of lipids requeriments in infants, children and adults. European J. Clin. Nutrition, v.53, n.1, p.66-67, 1999.

VICENTE, S. J.V. Formação de óxidos de colesterol em hambúrguer bovino em função do binômio tempo/ temperatura de preparação. 2003. 77p. [Dissertação de Mestrado. Faculdade de Saúde Pública. Universidade de São Paulo].

VICENTE, S.J.V.; TORRES, E.A.F.S. Formation of four cholesterol oxidation products and loss of free lipids, cholesterol ad water in beef hamburgers as a function of thermal processing. Food Control, v.18, n. p.63-68, 2007.

Recebido para publicação em 22 de junho de 2007 Aceito para publicação em 04 de agosto de 2008 